\title{
Prelmplantation Factor bolsters neuroprotection via modulating Protein Kinase A and Protein Kinase C signaling
}

\author{
M Mueller,1,2, A Schoeberlein ${ }^{3}$, J Zhou ${ }^{1,4}$, M Joerger-Messerli ${ }^{3}$, B Oppliger ${ }^{3}$, U Reinhart ${ }^{3}$, A Bordey ${ }^{5}$, D Surbek ${ }^{2,3}$, ER Barnea ${ }^{6,7}$, \\ Y Huang ${ }^{1}$ and $M$ Paidas ${ }^{1,8}$
}

A synthetic peptide (sPIF) analogous to the mammalian embryo-derived Prelmplantation Factor (PIF) enables neuroprotection in rodent models of experimental autoimmune encephalomyelitis and perinatal brain injury. The protective effects have been attributed, in part, to SPIF's ability to inhibit the biogenesis of microRNA let-7, which is released from injured cells during central nervous system (CNS) damage and induces neuronal death. Here, we uncover another novel mechanism of sPIF-mediated neuroprotection. Using a clinically relevant rat newborn brain injury model, we demonstrate that SPIF, when subcutaneously administrated, is able to reduce cell death, reverse neuronal loss and restore proper cortical architecture. We show, both in vivo and in vitro, that SPIF activates cyclic AMP dependent protein kinase (PKA) and calcium-dependent protein kinase (PKC) signaling, leading to increased phosphorylation of major neuroprotective substrates GAP-43, BAD and CREB. Phosphorylated CREB in turn facilitates expression of Gap43, Bdnf and Bcl2 known to have important roles in regulating neuronal growth, survival and remodeling. As is the case in SPIF-mediated let-7 repression, we provide evidence that sPIF-mediated PKA/PKC activation is dependent on TLR4 expression. Thus, we propose that SPIF imparts neuroprotection via multiple mechanisms at multiple levels downstream of TLR4. Given the recent FDA fast-track approval of SPIF for clinical trials, its potential clinical application for treating other CNS diseases can be envisioned.

Cell Death and Differentiation (2015) 22, 2078-2086; doi:10.1038/cdd.2015.55; published online 15 May 2015

Perinatal brain injury in the context of premature birth is a major cause of neonatal morbidity and mortality. ${ }^{1}$ Depending on the degree of prematurity, $15-20 \%$ of the affected newborns die during the postnatal period and $\sim 25 \%$ of survivors suffer significant long-term disability including cerebral palsy, epilepsy and increased hyperactivity. ${ }^{2}$ Therapeutic approaches to counteract the disastrous cascades of neonatal brain injury have been proposed. Unfortunately, in premature infants at risk, no neuroprotective agent has proven safe and effective so far. ${ }^{3}$

Secreted from developing embryos, Prelmplantation Factor (PIF) can be detected in the maternal circulation during pregnancy, ${ }^{4,5}$ and its presence has been correlated with live birth. ${ }^{5-7}$ PIF has been implicated in promoting embryo implantation through modulating maternal immune tolerance. ${ }^{5,8,9}$ Consistent with the immune function, a synthetic PIF analog (sPIF) of 15 amino acids (MVRIKPGSANKPSDD) that was subcutaneously administrated was able to both reverse and prevent paralysis through inhibiting neuroinflammation in a murine model of experimental autoimmune encephalomyelitis. ${ }^{10}$ The neuroprotective property of SPIF was further underscored by its ability to mitigate neuronal loss and microglial activation in a rat model of perinatal brain injury. ${ }^{11}$ The neuroprotective effects were attributed, at least in part, to SPIF's ability to downregulate microRNA let-7 in the injured brain. Abundantly expressed in the central nervous system (CNS), let-7 released from dying cells during brain injury induces neuronal death, exacerbating CNS damage. ${ }^{12,13}$ sPIF inhibited the biogenesis of let-7 in both neuronal and immune cells through Toll-like Receptor 4 (TLR4). ${ }^{11}$ As PIF imparts multitargeted effects, ${ }^{10}$ it is almost certain that inhibiting let-7 is not the only mechanism of PIF action.

In search of additional mechanisms, we chose to focus on cyclic AMP-dependent protein kinase (PKA) and calciumdependent protein kinase (PKC). PKA/PKC signaling is downstream of TLR4, ${ }^{14,15}$ and TLR4 was required for SPIFinduced neuroprotective effects. ${ }^{11}$ PKA/PKC are important signaling molecules in a variety of cellular functions, including cell growth and differentiation, neuronal plasticity and cellular

${ }^{1}$ Department of Obstetrics, Gynecology and Reproductive Sciences, Yale University School of Medicine, New Haven, CT, USA; ${ }^{2}$ Department of Obstetrics and Gynecology, University Hospital Bern, Bern, Switzerland; ${ }^{3}$ Department of Clinical Research, University of Bern, Bern, Switzerland; ${ }^{4}$ Department of Surgical Oncology, Affiliated Sir Run Run Shaw Hospital, Zhejiang University School of Medicine, Hangzhou, Zhejiang 310016, PR China; ${ }^{5}$ Department of Neurosurgery, Cellular and Molecular Physiology, Yale University School of Medicine, New Haven, CT, USA; ${ }^{6}$ Society for the Investigation of Early Pregnancy, Cherry Hill, NJ, USA; ${ }^{7}$ Biolncept LLC, Cherry Hill, NJ, USA and ${ }^{8}$ Women and Children's Center for Blood Disorders, Yale School of Medicine, New Haven, CT, USA

${ }^{*}$ Corresponding author: M Mueller, Department of Obstetrics, Gynecology and Reproductive Sciences, Yale University School of Medicine, 333 Cedar Street, FMB $339 \mathrm{~B}$, New Haven, CT 06520-8063, USA. Tel: +1 203737 1982; Fax: +1 203737 2327; E-mail: martin.muller@yale.edu

Abbreviations: PIF, Prelmplantation Factor; sPIF, synthetic PIF; PIFscr, scrambled PIF; CNS, central nervous system; PKA, cyclic AMP-dependent protein kinase; PKC, calcium-dependent protein kinase; TLR4, Toll-like Receptor 4; BAD, Bcl-associated death protein; GAP-43, Growth-associated protein 43; CREB, transcription factor cAMP-responsive element binding protein; LPS, lipopolysaccharides

Received 06.1.15; revised 26.3.15; accepted 09.4.15; Edited by M Freeman; published online 15.5.15 
response to hypoxia-ischemia. ${ }^{16-19}$ Mechanistically, PKA/ PKC activation leads to phosphorylation of serine and threonine residues on target proteins, thereby modulating protein stability, protein-protein interactions and catalytic activity. $^{20}$ In the case of brain injury, activation of the PKA/ PKC signaling pathways imparts neuroprotection by increasing expression of anti-apoptotic and neurotrophic molecules while reducing pro-apoptotic molecules in neurons. ${ }^{21-23}$ Not surprisingly, PKA/PKC pathways have been recognized as potent targets for neuroprotective strategies.

In the current study, we have examined and revealed a novel mechanism of PIF action. sPIF confers neuroprotection in a rat model of perinatal brain injury by modulating PKA/PKC signaling, which is recapitulated in vitro using neuronal cells. Overall, our data support clinical translation of sPIF treatment for hypoxic-ischemic brain injuries.

\section{Results}

SPIF promotes neuronal survival and migration in vivo. Given that SPIF induces global alterations in gene expression, it is highly likely that SPIF facilitates neuronal survival beyond targeting microRNA let-7.9,11 To evaluate the neuroprotective potential of SPIF, we induced brain injury in neonatal rats at P2 (Figure 1a). This time point matches ongoing neuronal development. ${ }^{24}$ The mammalian cerebral cortex is a six-layered structure comprising primarily pyramidal neurons and remaining inhibitory interneurons. ${ }^{25}$ The pyramidal neurons are generated in a well-defined inside-out manner, in which layer VI and V (deep layers) neurons arise and migrate first and layer IV-II (upper layers) neurons are born and migrate later. ${ }^{25}$ By birth, upper-layer neurons are still migrating until P7 whole deep-layer neurons are undergoing maturation. Thus, the major features of neuronal development and in particular deep-layer neurons may be altered as a consequence of perinatal brain injury. ${ }^{26,27}$

We evaluated cortical thickness (CT) first because in premature newborns reduced CT correlates with adverse neurological outcomes. ${ }^{28}$ We focused on evaluating regions containing motor and somatosensory cortices, as injury in these regions between the rhinal sulcus and the cingulum causes distinctive neuropathological alterations. ${ }^{11,27,29} \mathrm{We}$ examined the position of upper (CUX1-positive) and deeper (CUX1-negative) neurons in the cortex (Figure 1b). CUX1 is a well-defined molecular marker of upper-layer neurons (cortical layers II-IV). ${ }^{30}$ We detected reduced CT in deep cortical layers in the Injury compared with the Sham animals (Figure 1b). This observation confirmed previous reports that at P2 deep a

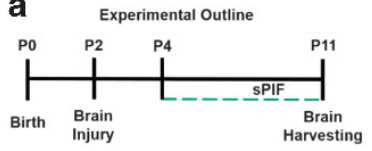

b
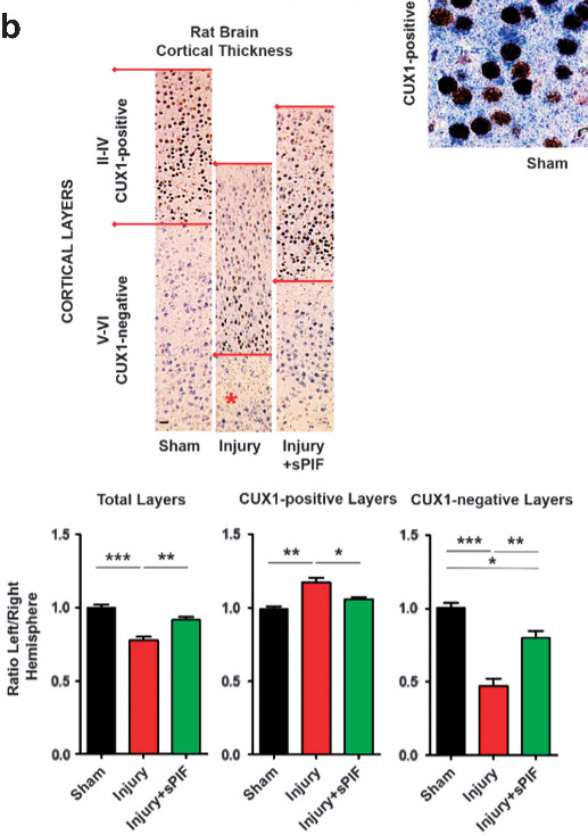

Rat Brain Cortical Architecture
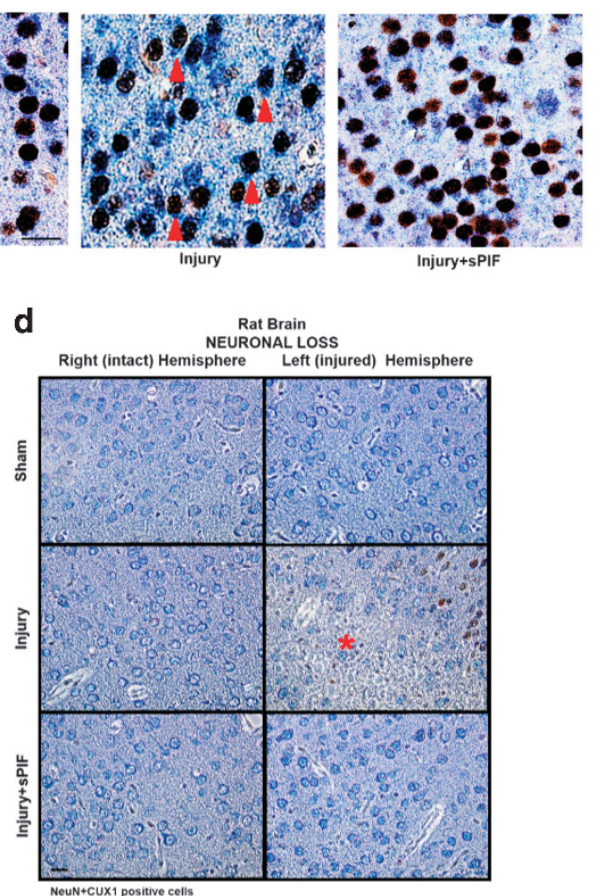

e

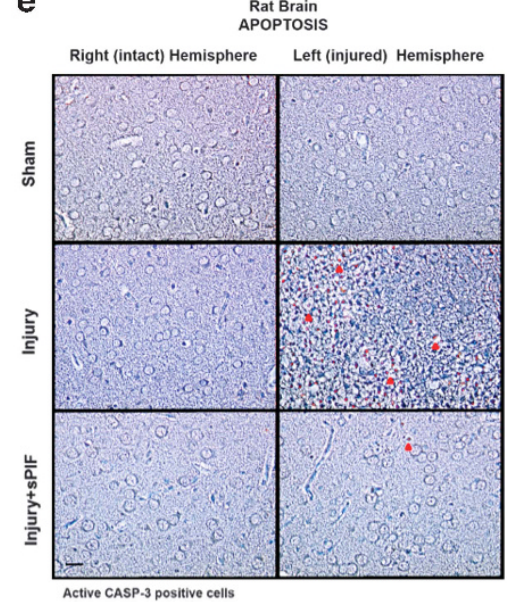

f

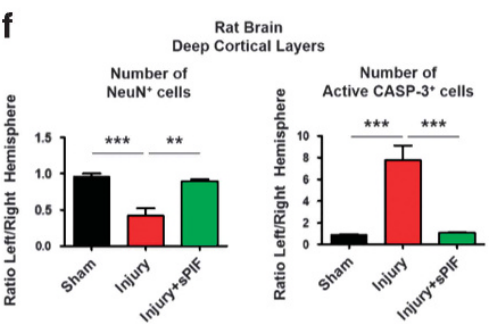

Figure 1 sPIF rescues cortical thickness and neuronal loss while decreasing active Caspase-3-positive cells in vivo. (a) Experimental outline. (b) Representative immunohistochemistry images containing somatosensory cortices and quantification of cortical thickness in upper (NeuN and CUX1-positive) and deeper (NeuN-positive and CUX1-negative) cortical layers. In injured animals, reduced cortical thickness is marked with a red star. (c) Representative immunohistochemistry images of CUX1-positive layers displaying cortical architecture. In injured animals, neurons display altered soma morphology (marked with red arrows). (d and e) Representative immunohistochemistry images stained with cresyl violet and (d) NeuN+CUX1 (for neuronal loss) and (e) active CASP-3 (for cell death) in deep cortical layers. In injured animals, neuronal loss is marked with a red star and active CASP-3-positive cells with red arrows. (f) Quantification of number of NeuN and active CASP-3-positive cells in deep cortical layers. Quantification results are displayed as ratio of the left (injured) to right (uninjured) hemisphere. Scale bar, $25 \mu \mathrm{m}$. Data are presented as mean \pm S.E.M. ( $n=4$ each group, one-way repeated measures ANOVA followed by Bonferroni's Multiple Comparison Test). ${ }^{*} P<0.05,{ }^{* *} P<0.01,{ }^{* * *} P<0.001$ 
cortical layers are particularly susceptible to hypoxic-ischemic brain injury. ${ }^{27,31}$ We also detected an expansion of the CUX1-positive layers as CUX1-positive cells were misplaced (Figure 1b). This observation suggested that hypoxicischemic injury at $\mathrm{P} 2$ resulted in disturbed neuronal migration of CUX1-positive cells. In addition, CUX1-positive cells displayed abnormal soma morphology as they exhibited a very thin leading process, which indicates disturbed neuronal migration (Figure 1c). ${ }^{32}$

Next, we analyzed the number of $\mathrm{NeuN}+$ (mature neurons) and active Caspase-3 (CASP-3, marker of apoptosis)positive cells in deep cortical layers, as injury predominantly reduced the thickness of these regions (Figure 1b). Brain injury at $P 2$ resulted in neuronal loss (Figures $1 d$ and f) and increased number of active CASP-3-positive cells (Figures 1e and f). Strikingly, in sPIF-treated animals (Injury+sPIF), both the CT (Figure 1b) and number of $\mathrm{NeuN}+$ neurons (Figures 1d and f) were restored (compare with Sham) with decreasing numbers of active CASP-3-positive cells (Figures 1e and f). Further, the cortical architecture and neuronal morphology in the sPIF-treated animals were comparable to those from the Sham animals (Figures $1 \mathrm{~b}$ and $\mathrm{c}$ ). Collectively, our results support the notion that sPIF acts to promote neuronal survival and preserve proper neuronal migration, thereby preserving the cortical architecture.

sPIF modulates PKA/PKC signaling in vivo. Given the neuroprotective effects of SPIF (Figure 1), we tested whether sPIF modulates PKA/PKC signaling. Having a screening approach in mind, we tested well-characterized S/T phosphorylation motif antibodies, which detect all phosphorylated PKA/PKC substrates in brain lysates. ${ }^{33}$ There is a global phosphorylation increase in the Injury compared with the Sham group (Figure 2a), which is in line with the notion that inflammatory and/or ischemic insults activate PKA/PKC signaling. ${ }^{34-36}$ Importantly, we detected a further increase in phosphorylation in the Injury+sPIF compared with the Injury group, suggesting possible modulation of PKA/PKC signaling by SPIF. $^{35}$ Thus, we reasoned that SPIF might enhance the phosphorylation of neuroprotective PKA/PKC substrates and therefore decided to test the phosphorylation status of BADSer112, GAP-43Ser41 and CREBSer133.

$\mathrm{Bcl}$-associated death protein (BAD) induces apoptosis; ${ }^{22,23}$ however, phosphorylation of BAD at the Ser112 site (BADSer112) results in liberation of anti-apoptotic proteins blocking apoptosis in vitro ${ }^{37,38}$ and in vivo. ${ }^{21,39}$ Growthassociated protein 43 (GAP-43) is a well-established marker of neuronal plasticity with increased expression during neuronal recovery. ${ }^{40,41}$ Importantly, phosphorylation at Ser41 regulates GAP-43 interaction with the cytoskeleton, promoting neurite outgrowth ${ }^{42}$ and dephosphorylation results in cytoskeleton destruction and growth cone collapse. ${ }^{43,44}$ The transcription factor CAMP-responsive element-binding protein (CREB) has been implicated in neuronal development, synaptic plasticity and in cell survival in response to ischemia. ${ }^{45,46}$ Phosphorylation of CREBSer133 leads to recruitment of CREB-binding protein $(\mathrm{CBP})$ at the promoter regions of CAMP-responsive genes including Gap43, Bcl2 and Bdnf, ${ }^{40,47,48}$ which impart neuroprotection. ${ }^{21,49,50}$ In summary, phosphorylation of all three proteins (BAD, GAP-43 and CREB) results in broad neuroprotective effects and represents a target for neuroprotective strategies.

Thus, we tested the three PKA/PKC substrates in our perinatal brain injury model. SPIF treatment indeed significantly enhanced phosphorylation of BADSer112, GAP-43Ser41 and CREBSer133 compared with the Injury animals (Figure 2b). The expressions of downstream genes $B c / 2, B d n f$ and Gap43 were expectedly increased in the sPIF-treated versus Injury animals (Figure 2c). Notably, we detected the increase in Gap43 at the RNA and protein levels (Figures $2 \mathrm{~b}$ and $\mathrm{c}$ ), which is consistent with PKA/PKCmediated neuronal recovery. ${ }^{40,41}$ On the other hand, we detected an increase in pro-apoptotic BAD protein and the RNA levels in Injury versus Sham and Injury+sPIF groups (Figures $2 b$ and $c$ ). This is in line with the notion of increased BAD protein and RNA levels in neurodegenerative diseases, ${ }^{51,52}$ which further supports the activity of SPIF of neuroprotection. Together, these results suggested that the neuroprotective effects observed (Figure 1) were at least in part because of modulation of PKA/PKC signaling, which prompted us to carry out more in-depth dissections of the underlying mechanisms in vitro.

\section{sPIF protects neuronal cells through a PKA/PKC-dependent} mechanism in vitro. We have previously used the murine neuroblastoma cell line N2a to demonstrate that SPIF inhibits the biogenesis of let-7, corroborating sPIF's ability of neuronal protection in vivo. ${ }^{11}$ To determine whether SPIF protects neuronal cells in part by regulating PKA/PKC signaling, N2a cells were pretreated with lipopolysaccharides (LPS; to mimic the in vivo situation ${ }^{11}$ ), and then subjected to SPIF or scrambled PIF (PIFscr, as a negative control). We detected a time-dependent increase in the PKA/PKC activity in SPIF- versus PIFscr-treated cells (Figure 3a), consistent with our in vivo results (Figures $2 \mathrm{~b}$ and $\mathrm{c}$ ). The ability of SPIF to reduce apoptosis and promote neuronal survival in $\mathrm{N} 2 \mathrm{a}$ was also evaluated. In line with our in vivo results (Figures $1 d-f$ and $2 c$ ), sPIF reduced the expression of the pro-apoptotic genes Bad and Casp3 while increasing that of the anti-apoptotic Bcl2 (Figure 3b). In addition, SPIF promoted neuronal survival as assessed by increased cell viability and reduced apoptosis (Figure $3 c$ ).

To confirm whether the SPIF-induced reduction of apoptosis was dependent on PKA/PKC signaling, pharmacological inhibitors specific against PKA (H89) and PKC (Gö6983) 53,54 were used. As shown in Figure 3c, sPIF-induced neuronal survival was abolished in the presence of $\mathrm{H} 89$ and Gö6983, consistent with a PKA/PKC-mediated mechanism. Expectedly, the presence of the inhibitors abrogated sPIF-induced phosphorylation increase of PKA/PKC substrates BADSer112, GAP-43Ser41 and CREBSer133 (Figures $3 d$ and e) as well as the increase in the expression of downstream targets Bcl2, Bdnf and Gap43 (Figure 3f). Taken together, our in vitro results lend a strong support to our in vivo findings that by modulating PKA/PKC signaling SPIF enhances the expression of neuroprotective genes while decreasing pro-apoptotic gene expression, thereby promoting neuronal survival. 


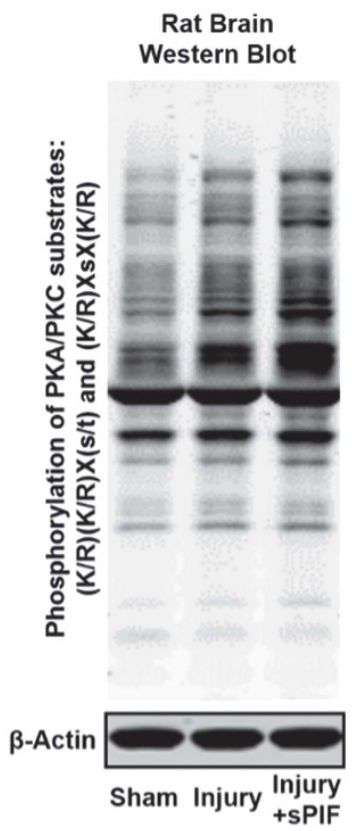

b

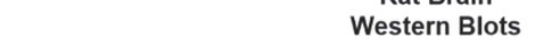

- Sham

$\square$ Injury

$\square$ Injury

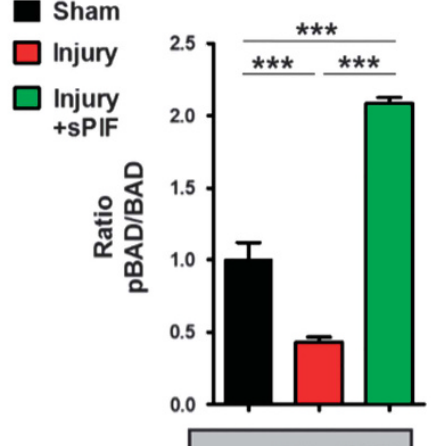

Rat Brain

Western Blots
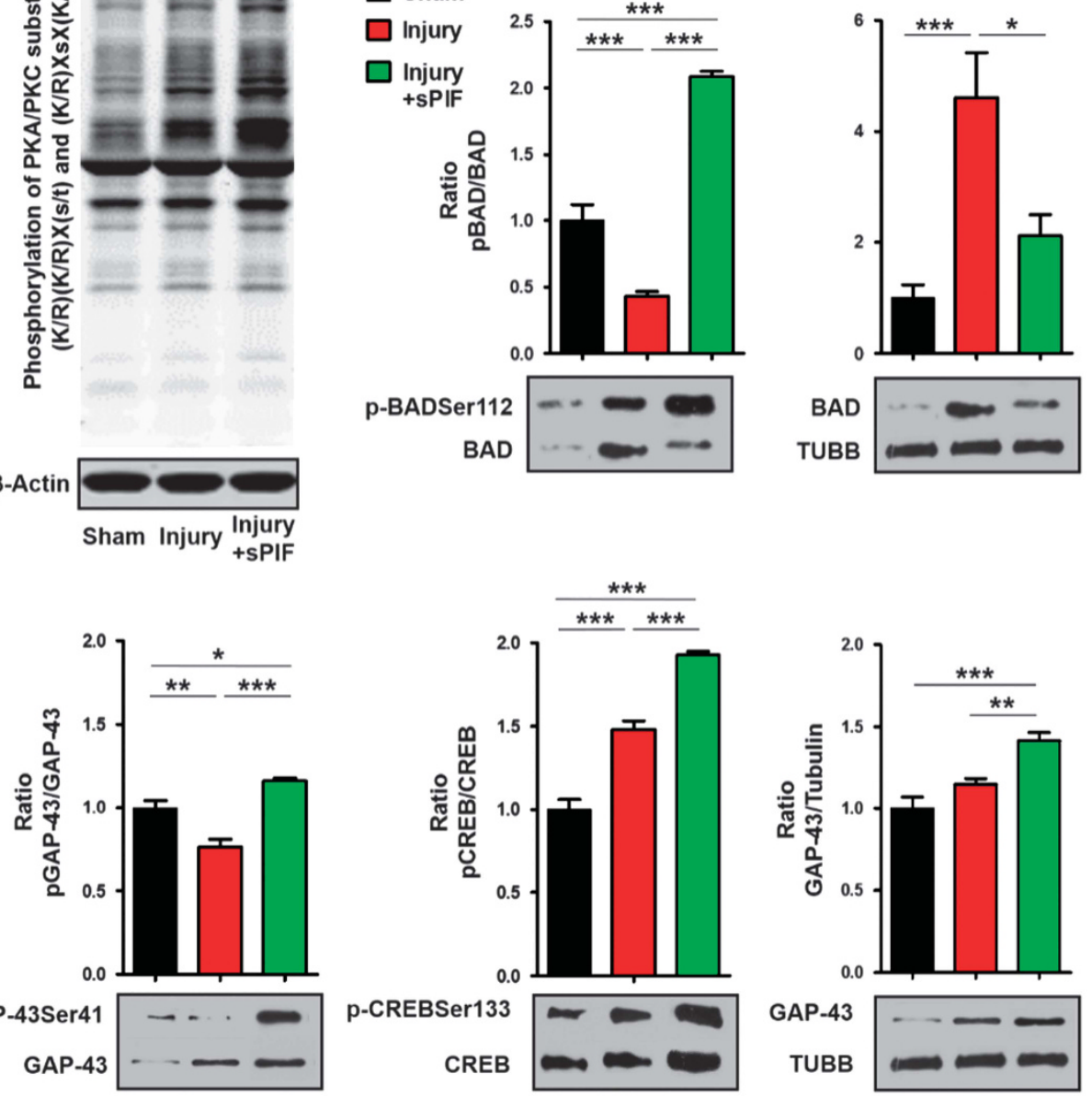

c

Rat Brain RT-qPCR
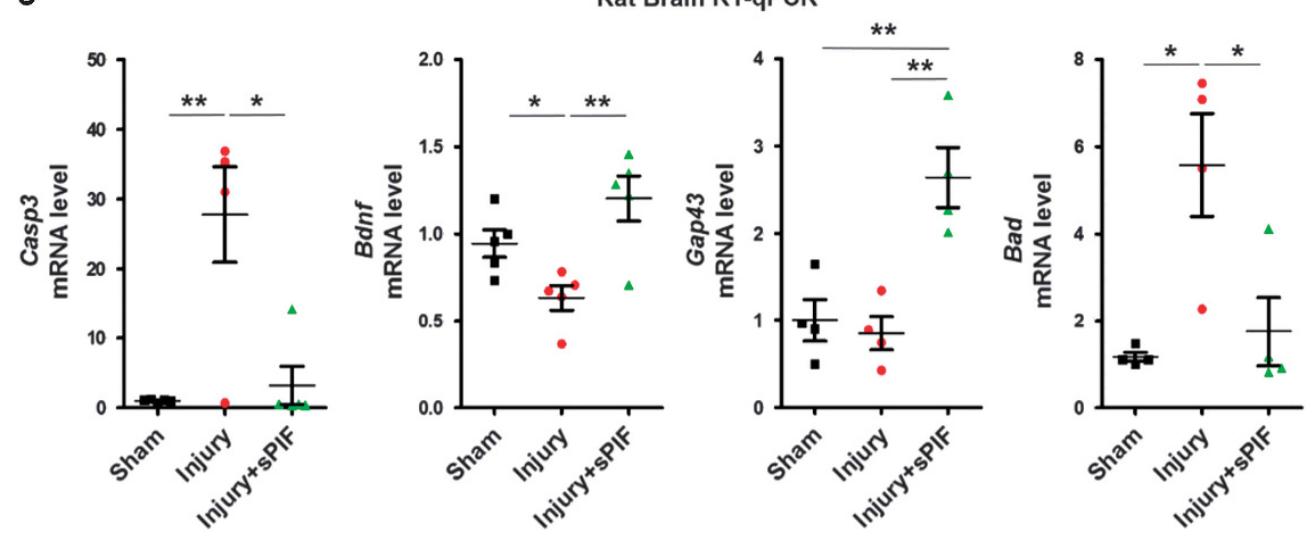

Figure 2 sPIF enhances phosphorylation of PKA/PKC substrates in vivo. (a) Representative western blots ( $n=3$ each group) of brain lysates probed with antibodies against specific $(K / R)(K / R) X(s / t)$ and $(K / R) X s X(K / R)$ phosphorylation motifs representing PKA/PKC substrates. $\beta$-Actin was used as a loading control. (b) Representative western blots ( $n=5$ each group) of brain lysates probed with specific antibodies against phosphorylated and total protein levels. Protein levels were quantified using a ratio of phosphorylated/ total protein level using beta-tubulin (TUBB) as a loading control. (c) RNAs were extracted from brain lysates ( $n=5$ each group) and levels determined using RT-qPCR. Quantification results of the Sham group were arbitrarily set as 1. Data are presented as mean \pm S.E.M. (one-way repeated measures ANOVA followed by Bonferroni's Multiple Comparison Test, two-tailed Student's t-test). ${ }^{*} P<0.05,{ }^{* *} P<0.01,{ }^{* \star *} P<0.001$ 
a

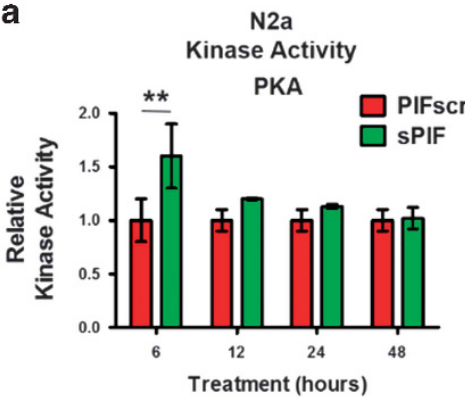

PKC

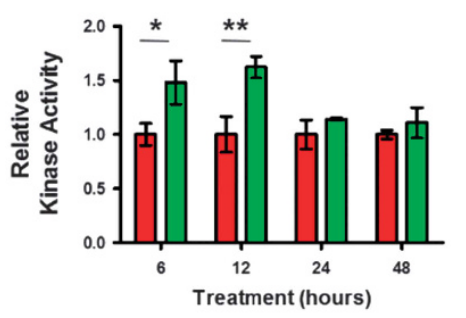

b

N2a
Cell Apoptosis
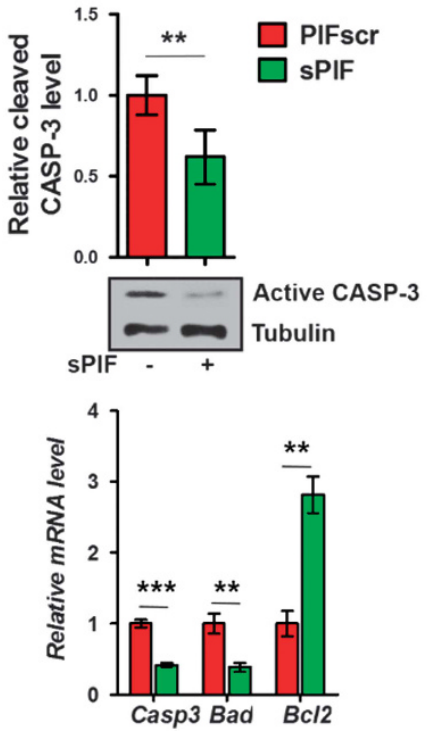<smiles></smiles>

Cell Survival
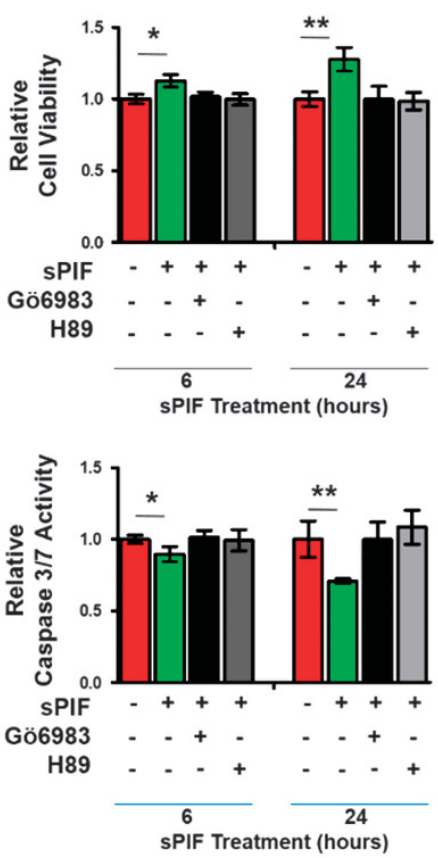

d

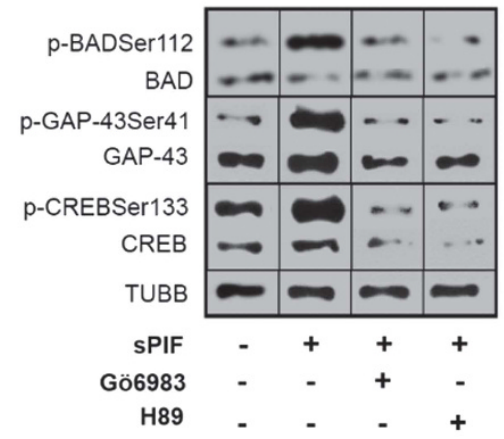

N2a
Western Blots

$f$
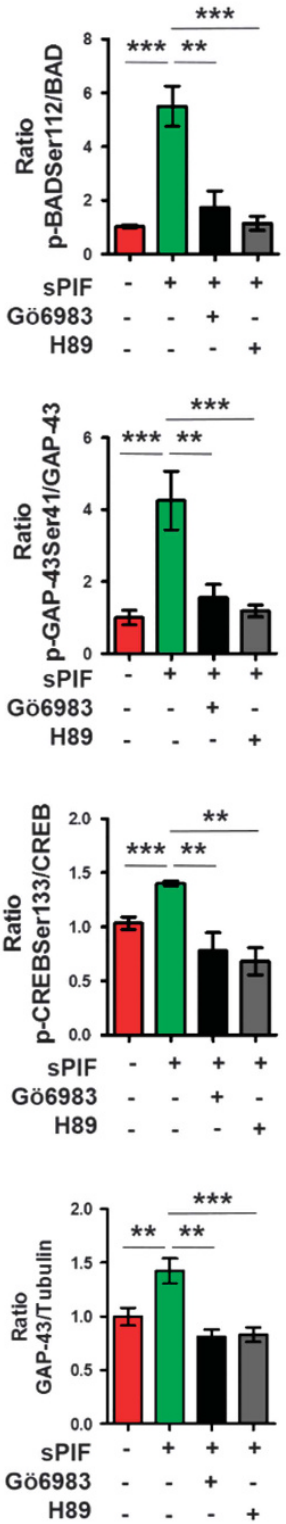
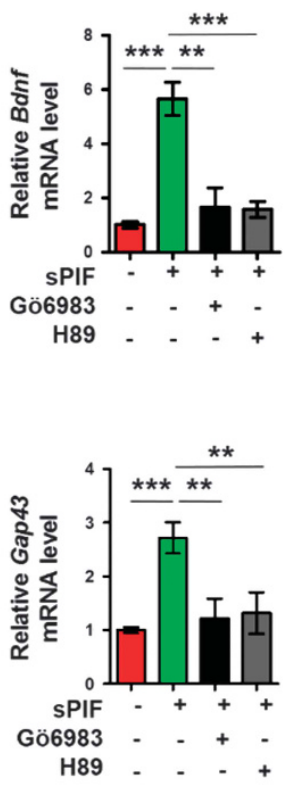

Figure 3 sPIF promotes neuronal survival in a PKA/PKC-dependent manner in vitro. N2a cells were incubated in the presence of LPS and on the next day treated with sPIF $(+)$ or PIFscr ( -$)$ at $200 \mathrm{nM}$ and/or in the presence of PKA (H89+) and PKC (Gö6983+) inhibitors. (a) Kinase activity assay was used to determine PKA and PKC activity at the indicated time points. (b) Protein and RNAs were extracted $24 \mathrm{~h}$ later and levels determined by western blot and RT-qPCR. (c) Cell viability and Caspase 3/7 activity were determined 6 and $24 \mathrm{~h}$ later. (d and e) Proteins were extracted $24 \mathrm{~h}$ later and levels determined with western blot analysis. (f) RNAs were extracted $24 \mathrm{~h}$ later and levels determined using RT-qPCR. Protein and RNA levels are presented after normalization using TUBB as loading control and against those from PIFscr-treated, which were arbitrarily set as 1 . All results are representative of three or more independent experiments. Data are presented as mean \pm S.D. (two-tailed Student's $t$-test). ${ }^{*} P<0.05$, ${ }^{* *} P<0.01$, ${ }^{* * *} P<0.001$

The sPIF-mediated PKA/PKC mechanism is TLR4dependent. TLR4 is known to be upstream of PKA/PKC signaling. ${ }^{14,15}$ We also have previously shown that TLR4 is required for SPIF-mediated let-7 inhibition in N2a cells. ${ }^{11}$ To determine whether sPIF-induced alteration in PKA/PKC signaling was also dependent on TLR4, TLR4 was knocked down in N2a using a TLR4-specific siRNA (siTLR4) $^{11}$ and effects on PKA/PKC substrate phosphorylation and downstream target gene expression were assessed. When TLR4 was downregulated (Figure 4a), sPIF-induced phosphorylation increase of PKA/PKC substrates BADSer112, GAP-43Ser41 and CREBSer133 was abolished (Figures $4 \mathrm{~b}$ and $\mathrm{c}$ ). Consistently, SPIF-induced increase in the expression of downstream target genes $\mathrm{Bcl}$, Bdnf and Gap43 was also negated (Figure 4d). Collectively, these results provide evidence that TLR4 is required for sPIF-mediated modulation of $\mathrm{PKA} / \mathrm{PKC}$ signaling. 
a

TLR4 Protein and mRNA

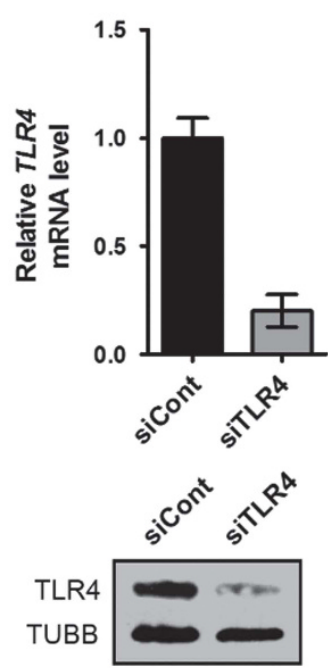

b

N2a

Western Blots

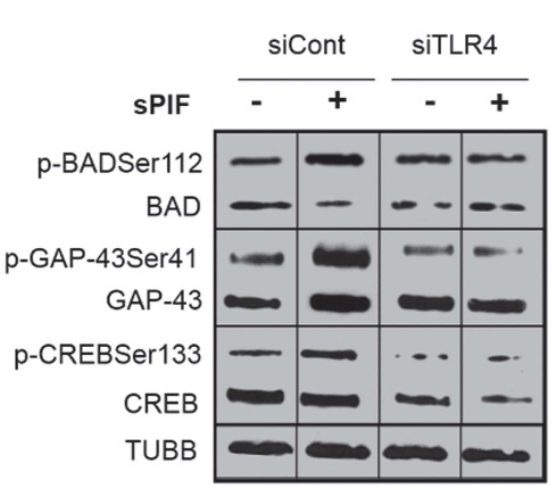

C
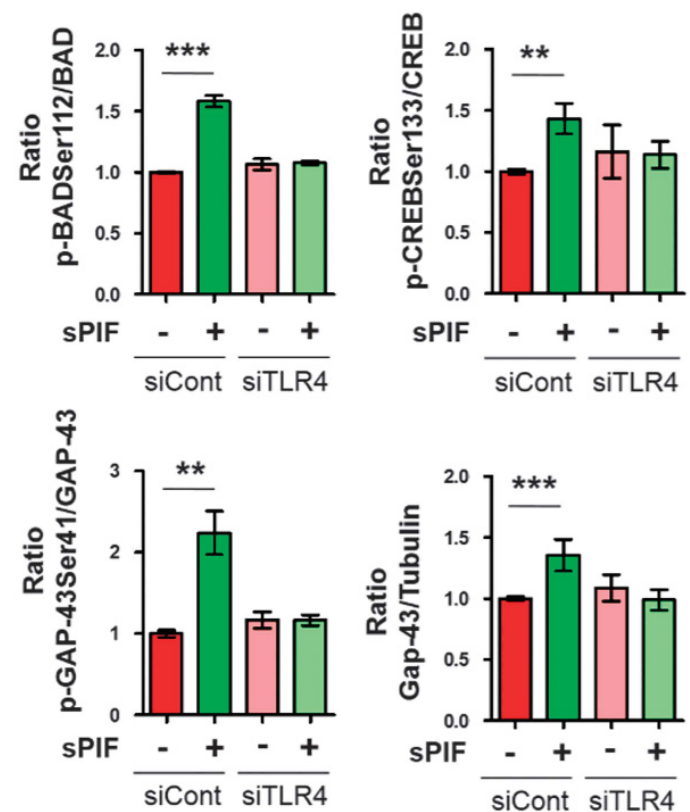

N2a

Western Blots

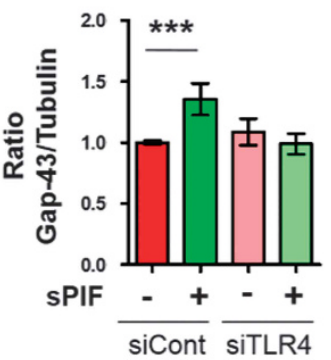

d
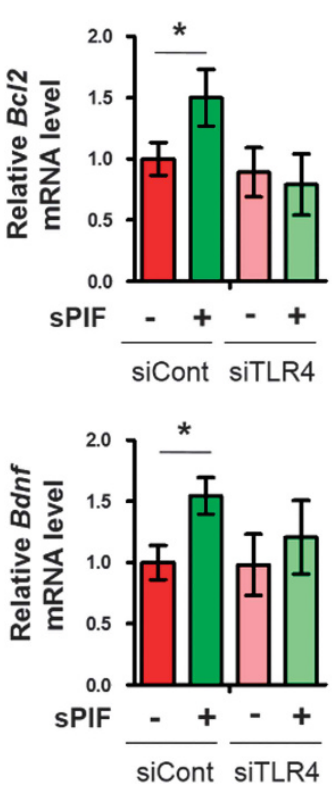

N2a

RT-qPCR

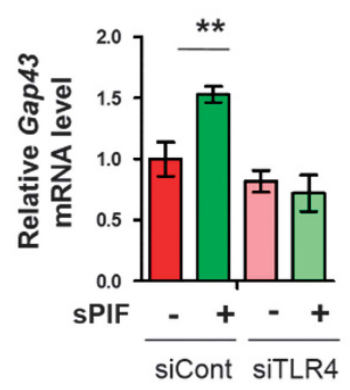

e

Proposed Model for sPIF-induced neuronal protection

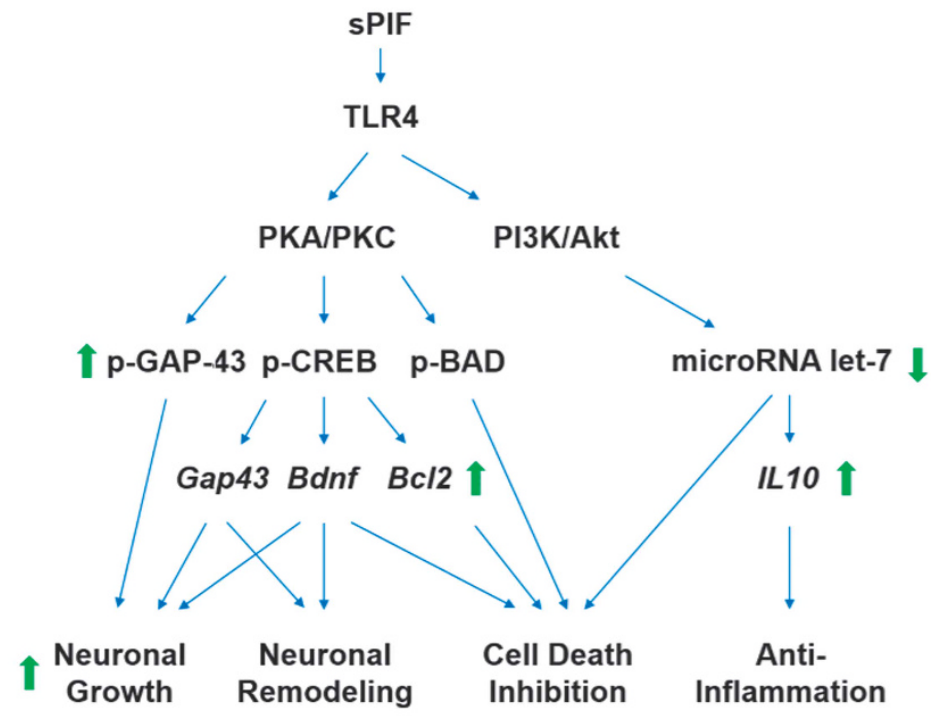

Figure 4 sPIF enhances phosphorylation of PKA/PKC substrates in a TLR4-dependent manner in vitro. N2a cells were transfected with control siRNA (siCon) or siRNA specific for TLR4 (siTLR4). On the following day, cells were pretreated with LPS and treated with sPIF $(+)$ or PIFscr $(-)$ at $200 \mathrm{nM}$. (a) RNAs and proteins were harvested $12 \mathrm{~h}$ post transfection and analyzed using RT-qPCR (upper) and western blot analysis (bottom), respectively. (b and c) Proteins were extracted $24 \mathrm{~h}$ later and levels determined using western blot analysis. (d) RNAs were extracted $24 \mathrm{~h}$ later and levels determined using RT-qPCR. (e) Proposed model for sPIF-induced neuronal protection. Protein and RNA levels are presented after normalization using TUBB as loading control and against those from PIFscr-treated, which were arbitrarily set as 1 . All results are representative of three or more independent experiments. Data are presented as mean \pm S.D. (two-tailed Student's $t$-test). ${ }^{\star} P<0.05,{ }^{* \star} P<0.01,{ }^{* \star \star} P<0.001$

\section{Discussion}

This report documents a new mechanism of sPIF-mediated neuroprotection. Using a clinically relevant newborn brain injury model, we demonstrate that subcutaneously injected SPIF was able to reduce cell death, reverse neuronal loss and restore proper cortical architecture. We provide evidence from both in vivo and in vitro studies that SPIF does so in part by modulating PKA/PKC signaling in a TLR4-dependent manner. As summarized in Figure 4e, sPIF activates PKA/PKC signaling through TLR4, resulting in phosphorylation of neuroprotective substrates GAP-43, CREB and BAD, which in turn stimulates expression of downstream genes Gap43, $B d n f$ and $B c / 2$. These genes have well-established roles in 
modulating neuronal growth, remodeling and survival. ${ }^{21,49,50}$ In light of our previous findings, ${ }^{11}$ SPIF downregulates microRNA let-7 expression through PI3K/Akt signaling, also in a TLR4-dependent manner reducing neuronal death. Meanwhile, decreased let-7 levels lead to increased expression of $I L 10$, a direct target of let-7-mediated inhibition ${ }^{55,56}$ and a potent anti-inflammatory cytokine in neuroprotection. ${ }^{57-59}$ Given the potent in vivo neuroprotective effects of SPIF, ${ }^{10,11}$ we propose that the two pathways (PKA/PKC and PI3K/Akt) may work synergistically in SPIF-mediated neuroprotection.

Our current and previous ${ }^{11}$ findings highlight an important role of TLR4 in SPIF-mediated neuroprotection, although the exact mechanism by which SPIF interacts with TLR4 remains to be investigated. The divergent in vivo effects of SPIF (neuroprotection, Figure 1) and LPS-induced neuroinflammation $^{24}$ suggest association/synergism with distinct cofactors of TLR4 signaling. ${ }^{35}$ Thus, it is conceivable that cofactors of SPIF likely act in concert to elicit/synergize downstream effects that are specific to SPIF but different from those induced by LPS. In addition, studies to determine in vivo effects of TLR4 silencing will be needed to firmly establish the role of TLR4 in SPIF-mediated neuroprotection.

With respect to potential clinical relevance and translation to human premature newborns, we decided to induce brain injury by a combination of LPS and hypoxia-ischemia at P2 and started therapy at P4 (Figure 1a) in neonatal rats. We used LPS before hypoxia-ischemia because perinatal infection/ inflammation contributes substantially to cerebral injury in immature neonates. ${ }^{60}$ In neonatal rats, LPS results in timeand TLR4-dependent sensitization to the following injury, ${ }^{61,62}$ which mimics the situation in human newborns when infection/ inflammation occurs. In addition, we induced brain injury at P2 because neonatal rats correspond to human immature infants in terms of brain development and susceptibility to injury. ${ }^{24}$ It is also noteworthy that SPIF promotes neuroprotection despite the late starting point (P4) of the therapy. From a clinical point of view this late treatment is promising, as in immature infants the precise timing of the injury often remains unclear (whether it occurs pre-, during labor or during the postnatal period) and treatment options frequently result in no benefit. ${ }^{63}$

Finally, our finding that SPIF targets PKA/PKC is particularly intriguing as studies have pointed to the modulation of PKA/ PKC signaling as a therapeutic approach for adult neuronal injury such as Alzheimer's Disease and cerebral ischemic/ stroke. ${ }^{20,64,65}$ Importantly, in our previous studies we have demonstrated that subcutaneously injected SPIF was able to reach the brain and localize to neuronal cells as assessed using immunofluorescence. ${ }^{11}$ Furthermore, in this previous study, we showed that such a dosing regimen was indeed effective in rescuing brain damage in the same rat model used in this current study. Together, the current and previously reported neuroprotective evidence, ${ }^{10,11}$ combined with the FDA fast-track approval and recently started Phase I Clinical Trial for autoimmune hepatitis (https://clinicaltrials.gov), supports the potential clinical application of SPIF for both perinatal and adult brain injuries.

Materials and Methods

Cell culture. Mouse neuroblastoma cells (N2a) were purchased from ATCC. Cells were maintained in DMEM (Gibco, Life Technologies, Carlsbad, CA, USA;
11965-092) supplemented with 10\% FBS (Gibco, 16140-071), $1 \mathrm{mM} \mathrm{L-glutamine}$ (Gibco, 25030-081) and 1\% penicillin-streptomycin (Gibco, 15140-122) in $5 \% \mathrm{CO}_{2}$ atmosphere at $37^{\circ} \mathrm{C}$. Trypsin-EDTA (0.25\%, Gibco, 25200-056) was applied to detach N2a from culture plates.

Treatment of cultured cells. Cells were seeded in 48-well plates in regular growth media at a density of $\sim 8 \times 10^{4}$ per well. The next day, media were replaced with free-serum media containing LPS $(0.5 \mu \mathrm{g} / \mathrm{ml})$ for $24 \mathrm{~h}$. The day after, media were replaced with serum-free media containing sPIF or PIFscr at $200 \mathrm{nM}$ concentration, followed by incubation for the indicated time duration. For PKA/PKC inhibitor studies after LPS pretreatment, media were replaced with serum-free media containing inhibitors for PKA (H89) or PKC (Gö6983) at $5 \mu \mathrm{M}$ concentration. After 30 min, media were replaced with serum-free media containing PKA or PKC inhibitor and SPIF or PIFscr at $200 \mathrm{nM}$ for the indicated time duration.

Antibodies, siRNAs, inhibitors and peptides. See Supplementary Information for details.

TLR4 siRNA knockdown. See Supplementary Information for details.

Western blot analyses and qRT-PCR. See Supplementary Information for details.

Cell viability and apoptosis (Caspase $3 / 7$ ) activity. Briefly, cells were seeded in 96-well plates at a density of $1 \times 10^{4} /$ well. The next day, media were replaced with serum-free media containing LPS $(0.5 \mu \mathrm{g} / \mathrm{ml})$ for $24 \mathrm{~h}$. The next day, media were replaced with serum-free media containing sPIF or PIFscr at $200 \mathrm{nM}$ concentration. Cell viability and apoptosis (caspase 3/7 activity) were measured 6 and $24 \mathrm{~h}$ post sPIF or PIFscr treatment using the Cell Titer Blue Cell Viability kit (Promega, Madison, WI, USA; no. G8081) and the Apo-ONE Homogeneous Caspase-3/7 Assay kit (Promega, no. G7791), respectively.

PKA and PKC activity. Briefly, cells were seeded in 48-well plates at a density of $8 \times 10^{4} /$ well. The next day, media were replaced with serum-free media containing LPS $(0.5 \mu \mathrm{g} / \mathrm{ml})$ for $24 \mathrm{~h}$. The next day, media were replaced with serumfree media containing SPIF or PIFscr at $200 \mathrm{nM}$ concentration. PKA and PKC activities were measured at $6,12,24$ and $48 \mathrm{~h}$ post-sPIF and -PIFscr treatment using the PKA (Enzo Life Sciences, Farmingdale, NY, USA; ADi-EKS-390 A) and PKC (Enzo Life Sciences, ADi-EKS-420A) kinase activity kit, respectively, according to the manufacturer's protocols.

Induction of hypoxic-ischemic brain injury. All animal procedures and perioperative care were approved by the Ethics Committee and Veterinary Department of the Canton of Berne, Switzerland. Animals (36 neonatal Wistar rats) were randomly divided into three groups (Sham, Injury and Injury+SPIF; $n=12$ each group). Injury and Injury+SPIF groups received LPS (0.1 mg/kg BW, i.p.) at postnatal day 1 (P1). On the following day, the animals were placed on an approved thermoregulatory device $\left(37^{\circ} \mathrm{C}\right)$, anesthetized using isoflurane and a $5-\mathrm{mm}$ jugular skin incision was made (using microscissors). We exposed the left carotid artery, separated from the nervus vagus and permanently ligated (6.0 prolene suture) and cut the artery. ${ }^{11,66}$ We closed the skin incision using a 6-0 prolene suture. The complete procedure did not exceed $10 \mathrm{~min}$. Animals were returned to the mother for a recovery period of $60 \mathrm{~min}$ and then placed in a gas-tight cylinder, which was flooded with pre-warmth and humidified gas mixture $\left(8 \% \mathrm{O}_{2}\right.$ and $\left.92 \% \mathrm{~N}_{2}\right)$ for a period of up to $65 \mathrm{~min}$, thus creating a stable $8 \% \mathrm{O}_{2}$ environment. Starting on postnatal day 4, the Injury group received phosphate-buffered saline (Vehicle) and the Injury+sPIF group received SPIF ( $0.75 \mathrm{mg} / \mathrm{kg}$ BW twice daily) until brain harvesting on postnatal day 11. The Sham group consisted of sham-operated animals $(0.9 \% \mathrm{NaCl}$ instead of LPS, exposure of the carotid artery without ligation, no hypoxia).

Perioperative care. See Supplementary Information for details.

Immunohistochemistry. See Supplementary Information for details.

Quantitative assessment of the brain. Assessment of neuronal loss and number of active CASP-3-positive cells was made in the region of interest (ROI) defined as the deep cerebral cortex (cortical layers V and VI in Figure. 108 according to a stereotactic rat atlas) ${ }^{67}$ and in each hemisphere independently. This 
cortical area between the rhinal sulcus and the cingulum contains the cerebral white matter, and hypoxia-ischemia on P3 was reported to cause distinctive neuropathological alterations in these regions. ${ }^{27,29} \mathrm{CT}$, and CUX1-positive and -negative CT were determined in coronal sections (Figure 108 according to a stereotactic rat atlas) ${ }^{67}$ containing motor and somatosensory cortices. The sections were stained with cresyl violet and evaluation performed in each hemisphere independently. Because of the variability in the thickness of the motor cortex, we drew a perpendicular line to the white matter through the six layers of the cortex to measure the thickness. All images of immunohistochemical stainings were obtained with a BX51 microscope (Olympus, Tokyo, Japan) equipped with a digital camera and images acquired using a $\times 40$ objective images for $\mathrm{ROI}$ evaluation and $\mathrm{a} \times 10$ objective for CT and Neuronal Displacement evaluation. An independent observer acquired six consecutive coronal sections per hemisphere and animal for each specific immunostaining blinded to the experimental conditions. Images were analyzed and reconstructed using Image $J$ (US National Institutes of Health, Bethesda, MD, USA).

Quantification and statistical analysis. All quantifications such as manual cell counts were performed in a blinded manner. Positive cell counts for NeuN and active CASP-3-positive cells were performed in the ROI (see above) as previously reported. ${ }^{27,68,69}$ Number of cells was determined by unbiased counting of positive cells. ${ }^{68}$

Data analysis. We avoided variability resulting from tissue handling and staining, and interanimal developmental variations by using a ratio of left (ipsilateral to carotid occlusion) to the right hemisphere. ${ }^{29,69}$ Data from in vivo studies are represented as mean \pm S.E.M. Data from in vitro studies are represented as mean \pm S.D. Single comparisons with control were made using two-tailed Student's $t$-test or Mann-Whitney test. We used one-way repeated measures ANOVA followed by Bonferroni's Multiple Comparison Test for multigroup design. $P<0.05$ was considered to be statistically significant. Data handling and statistical processing were performed using the Microsoft Excel and GraphPad Prism Software.

\section{Conflict of Interest}

ERB is CSO of Biolncept, LLC, NJ, USA. The remaining authors declare no conflict of inerest.

Acknowledgements. This work was supported by funds from the Eagle Foundation (Switzerland), Cryo-Save AG (Switzerland), Forschungs-Grant (University Hospital Bern, Switzerland) and unrestricted fund from Biolncept LLC. We thank Jeff Silva, Cell Signaling Technology, MA, USA for performing the PKA/PKC motif. antibodies experiments. We thank Cedric Simillion, University of Bern, for support with the statistical analysis.

1. Preterm birth: crisis and opportunity. Lancet 2006; $368: 339$

2. Volpe JJ. Brain injury in premature infants: a complex amalgam of destructive and developmental disturbances. Lancet Neurol 2009; 8: 110-124.

3. Higgins RD, Raju T, Edwards AD, Azzopardi DV, Bose CL, Clark RH et al. Hypothermia and other treatment options for neonatal encephalopathy: an executive summary of the Eunice Kennedy Shriver NICHD workshop. J Pediatr 2011; 159: 851-858.

4. Barnea ER. Insight into early pregnancy events: the emerging role of the embryo. Am J Reprod Immunol 2004; 51: 319.

5. Barnea ER, Kirk D, Ramu S, Rivnay B, Roussev R, Paidas MJ. Prelmplantation Factor (PIF) orchestrates systemic antiinflammatory response by immune cells: effect on periphera blood mononuclear cells. Am J Obstet Gynecol 2012; 207: 313.

6. Stamatkin CW, Roussev RG, Stout M, Absalon-Medina V, Ramu S, Goodman C et al. Prelmplantation Factor (PIF) correlates with early mammalian embryo development-bovine and murine models. Reprod Biol Endocrinol 2011; 9: 63.

7. Ramu S, Stamatkin C, Timms L, Ruble M, Roussev RG, Barnea ER. Prelmplantation factor (PIF) detection in maternal circulation in early pregnancy correlates with live birth (bovine model). Reprod Biol Endocrinol 2013; 11: 105.

8. Roussev RG, Dons'koi BV, Stamatkin C, Ramu S, Chernyshov VP, Coulam CB et al. Preimplantation factor inhibits circulating natural killer cell cytotoxicity and reduces $\mathrm{CD69}$ expression: implications for recurrent pregnancy loss therapy. Reprod Biomed Online 2013; 26: 79-87.

9. Paidas MJ, Krikun G, Huang SJ, Jones R, Romano M, Annunziato $\mathrm{J}$ et al. A genomic and proteomic investigation of the impact of preimplantation factor on human decidual cells. Am J Obstet Gynecol 2010; 202: 459 e451-459 e 458.
10. Weiss L, Or R, Jones RC, Amunugama R, JeBailey L, Ramu $S$ et al. Preimplantation factor $\left(\mathrm{PIF}^{*}\right)$ reverses neuroinflammation while promoting neural repair in EAE model. J Neurol Sci 2012; 312: 146-157.

11. Mueller M, Zhou J, Yang L, Gao Y, Wu F, Schoeberlein A et al. Prelmplantation factor promotes neuroprotection by targeting microRNA let-7. Proc Natl Acad Sci USA 2014; 111: 13882-13887.

12. Lehmann SM, Kruger C, Park B, Derkow K, Rosenberger $\mathrm{K}$, Baumgart $\mathrm{J}$ et al. An unconventional role for miRNA: let-7 activates Toll-like receptor 7 and causes neurodegeneration. Nat Neurosci 2012; 15: 827-835.

13. Selvamani A, Sathyan P, Miranda RC, Sohrabji F. An antagomir to microRNA Let7f promotes neuroprotection in an ischemic stroke model. PLOS ONE 2012; 7: 332662

14. Hou L, Wang X. PKC and PKA, but not PKG mediate LPS-induced CGRP release and $[\mathrm{Ca}(2+)]$ (i) elevation in DRG neurons of neonatal rats. J Neurosci Res 2001; 66: $592-600$

15. Tse KH, Chow KB, Leung WK, Wong YH, Wise H. Primary sensory neurons regulate Toll-like receptor-4-dependent activity of glial cells in dorsal root ganglia. Neuroscience 2014; 279: 10-22.

16. Leenders AG, Sheng ZH. Modulation of neurotransmitter release by the second messengeractivated protein kinases: implications for presynaptic plasticity. Pharmacol Ther 2005; 105: 69-84.

17. Libien J, Sacktor TC, Kass IS. Magnesium blocks the loss of protein kinase C, leads to a transient translocation of PKC(alpha) and PKC(epsilon), and improves recovery after anoxia in rat hippocampal slices. Brain Res Mol Brain Res 2005; 136: 104-111.

18. Raval AP, Dave KR, Mochly-Rosen D, Sick TJ, Perez-Pinzon MA. Epsilon PKC is required for the induction of tolerance by ischemic and NMDA-mediated preconditioning in the organotypic hippocampal slice. J Neurosci 2003; 23: 384-391.

19. Selvatici R, Melloni E, Ferrati M, Piubello C, Marincola FC, Gandini E. Adaptative value of a PKC-PKI55 feedback loop of inhibition that prevents the kinase's deregulation. J Mol Evol 2003; 57 : 131-139.

20. Sun MK, Alkon DL. Pharmacology of protein kinase $\mathrm{C}$ activators: cognition-enhancing and antidementic therapeutics. Pharmacol Ther 2010; 127: 66-77.

21. Chen Z, Jalabi W, Hu W, Park HJ, Gale JT, Kidd GJ et al. Microglial displacement of inhibitory synapses provides neuroprotection in the adult brain. Nat Commun 2014; 5: 4486.

22. Park HJ, Park KH, Shin KS, Lee MK. The roles of cyclic AMP-ERK-Bad signaling pathways on 6-hydroxydopamine-induced cell survival and death in PC12 cells. Toxicology 2013; 27: 2233-2241.

23. Zhao H, Yenari MA, Cheng D, Sapolsky RM, Steinberg GK. Bcl-2 overexpression protects against neuron loss within the ischemic margin following experimental stroke and inhibits cytochrome c translocation and caspase-3 activity. J Neurochem 2003; 85: 1026-1036.

24. Salmaso N, Jablonska B, Scafidi J, Vaccarino FM, Gallo V. Neurobiology of premature brain injury. Nat Neurosci 2014; 17: 341-346.

25. Molyneaux BJ, Arlotta P, Menezes JR, Macklis JD. Neuronal subtype specification in the cerebral cortex. Nat Rev Neurosci 2007; 8: 427-437.

26. Ortinau C, Neil J. The neuroanatomy of prematurity: Normal brain development and the impact of preterm birth. Clin Anat 2014; 28: 168-183.

27. Sizonenko SV, Kiss JZ, Inder T, Gluckman PD, Williams CE. Distinctive neuropathologic alterations in the deep layers of the parietal cortex after moderate ischemic-hypoxic injury in the P3 immature rat brain. Pediatr Res 2005; 57: 865-872.

28. Bjuland KJ, Lohaugen GC, Martinussen M, Skranes J. Cortical thickness and cognition in very-low-birth-weight late teenagers. Early Hum Dev 2013; 89: 371-380.

29. Sizonenko SV, Sirimanne E, Mayall Y, Gluckman PD, Inder T, Williams C. Selective cortical alteration after hypoxic-ischemic injury in the very immature rat brain. Pediatr Res 2003; 54 263-269.

30. Ferrere A, Vitalis T, Gingras H, Gaspar P, Cases O. Expression of Cux-1 and Cux-2 in the developing somatosensory cortex of normal and barrel-defective mice. Anat Rec A Discov Mol Cell Evol Biol 2006; 288: 158-165

31. Kida H, Nomura S, Shinoyama M, Ideguchi M, Owada Y, Suzuki M. The effect of hypothermia therapy on cortical laminar disruption following ischemic injury in neonatal mice. PLOS ONE 2013; 8: e68877.

32. Reiner O, Sapir T. Cdk5 checks p27kip 1 in neuronal migration. Nat Cell Biol 2006: 8: 11-13.

33. Wang IT, Allen M, Goffin D, Zhu X, Fairless AH, Brodkin ES et al. Loss of CDKL5 disrupts kinome profile and event-related potentials leading to autistic-like phenotypes in mice. Proc Natl Acad Sci USA 2012; 109: 21516-21521.

34. Kitagawa K. CREB and CAMP response element-mediated gene expression in the ischemic brain. FEBS J 2007; 274: 3210-3217.

35. Goldsmith M, Avni D, Ernst O, Glucksam Y, Levy-Rimler G, Meijler MM et al. Synergistic IL-10 induction by LPS and the ceramide-1-phosphate analog PCERA-1 is mediated by the cAMP and p38 MAP kinase pathways. Mol Immunol 2009; 46: 1979-1987.

36. Cheung HH, Teves L, Wallace MC, Gurd JW. Increased phosphorylation of the NR1 subunit of the NMDA receptor following cerebral ischemia. J Neurochem 2001; 78: 1179-1182.

37. Chen J, Liu Y, Soh JW, Aguilera G. Antiapoptotic effects of vasopressin in the neuronal cell line H32 involve protein kinase Calpha and beta. J Neurochem 2009; 110: 1310-1320.

38. Harada H, Becknell B, Wilm M, Mann M, Huang LJ, Taylor SS et al. Phosphorylation and inactivation of BAD by mitochondria-anchored protein kinase A. Mol Cell 1999; 3: 413-422.

39. Li Y, Zeng M, Chen W, Liu C, Wang F, Han X et al. Dexmedetomidine reduces isofluraneinduced neuroapoptosis partly by preserving PI3K/Akt pathway in the hippocampus of neonatal rats. PLOS ONE 2014; 9: e93639. 
40. Lai HC, Wu MJ, Chen PY, Sheu TT, Chiu SP, Lin MH et al. Neurotrophic effect of citrus 5hydroxy-3,6,7,8,3',4'-hexamethoxyflavone: promotion of neurite outgrowth via CAMP/PKA/ CREB pathway in PC12 cells. PLOS ONE 2011; 6: e28280.

41. Das KP, Freudenrich TM, Mundy WR. Assessment of PC12 cell differentiation and neurite growth: a comparison of morphological and neurochemical measures. Neurotoxicol Teratol 2004; 26: 397-406.

42. Korshunova I, Novitskaya V, Kiryushko D, Pedersen N, Kolkova K, Kropotova E et al. GAP-43 regulates NCAM-180-mediated neurite outgrowth. J Neurochem 2007; 100: 1599-1612.

43. Strittmatter SM, Valenzuela D, Kennedy TE, Neer EJ, Fishman MC. G0 is a major growth cone protein subject to regulation by GAP-43. Nature 1990; 344: 836-841.

44. Huang KP, Huang FL, Chen HC. Hypoxia/ischemia induces dephosphorylation of rat brain neuromodulin/GAP-43 in vivo. J Neurochem 1999; 72: 1294-1306.

45. Finkbeiner S. CREB couples neurotrophin signals to survival messages. Neuron 2000; 25: $11-14$.

46. Mayr BM, Canettieri G, Montminy MR. Distinct effects of CAMP and mitogenic signals on CREB-binding protein recruitment impart specificity to target gene activation via CREB. Proc Natl Acad Sci USA 2001; 98: 10936-10941.

47. Tardito D, Perez J, Tiraboschi E, Musazzi L, Racagni G, Popoli M. Signaling pathways regulating gene expression, neuroplasticity, and neurotrophic mechanisms in the action of antidepressants: a critical overview. Pharmacol Rev 2006; 58: 115-134.

48. Meller R, Minami M, Cameron JA, Impey S, Chen D, Lan JQ et al. CREB-mediated Bcl-2 protein expression after ischemic preconditioning. J Cereb Blood Flow Metab 2005; 25: 234-246.

49. Zhang $\mathrm{L}$, Zhao $\mathrm{H}$, Zhang $X$, Chen $\mathrm{L}$, Zhao $X$, Bai $X$ et al. Nobiletin protects against cerebral ischemia via activating the $\mathrm{p}$-Akt, $\mathrm{p}$-CREB, BDNF and Bcl-2 pathway and ameliorating BBB permeability in rat. Brain Res Bull 2013; 96: 45-53.

50. Sist B, Fouad K, Winship IR. Plasticity beyond peri-infarct cortex: spinal up regulation of structural plasticity, neurotrophins, and inflammatory cytokines during recovery from cortical stroke. Exp Neurol 2014; 252: 47-56.

51. Kim HW, Chang YC, Chen M, Rapoport SI, Rao JS. Chronic NMDA administration to rats increases brain pro-apoptotic factors while decreasing anti-Apoptotic factors and causes cell death. BMC Neurosci 2009; 10: 123.

52. Shacka JJ, Roth KA. Bcl-2 family and the central nervous system: from rheostat to real complex. Cell Death Differ 2006; 13: 1299-1304.

53. Kaddour H, Hamdi Y, Vaudry D, Basille M, Desrues L, Leprince J et al. The octadecaneuropeptide ODN prevents 6-hydroxydopamine-induced apoptosis of cerebellar granule neurons through a PKC-MAPK-dependent pathway. J Neurochem 2013; 125: 620-633.

54. Talman V, Amadio M, Osera C, Sorvari S, Boije Af Gennas G, Yli-Kauhaluoma J et al. The C1 domain-targeted isophthalate derivative $\mathrm{HMl}-1 \mathrm{~b} 11$ promotes neurite outgrowth and GAP-43 expression through PKCalpha activation in SH-SY5Y cells. Pharmacol Res 2013; 73: $44-54$.
55. Schulte LN, Eulalio A, Mollenkopf HJ, Reinhardt R, Vogel J. Analysis of the host microRNA response to Salmonella uncovers the control of major cytokines by the let-7 family. EMBO J 2011; 30: 1977-1989.

56. Swaminathan S, Suzuki K, Seddiki N, Kaplan W, Cowley MJ, Hood CL et al. Differential regulation of the Let-7 family of microRNAs in CD4+ T cells alters IL-10 expression. J Immunol 2012; 188: 6238-6246.

57. Ooboshi H, Ibayashi S, Shichita T, Kumai Y, Takada J, Ago T et al. Postischemic gene transfer of interleukin-10 protects against both focal and global brain ischemia. Circulation 2005; 111: 913-919.

58. Sharma S, Yang B, Xi X, Grotta JC, Aronowski J, Savitz SI. IL-10 directly protects cortical neurons by activating PI-3 kinase and STAT-3 pathways. Brain Res 2011; 1373: 189-194.

59. Grilli M, Barbieri I, Basudev H, Brusa R, Casati C, Lozza G et al. Interleukin-10 modulates neuronal threshold of vulnerability to ischaemic damage. Eur J Neurosci 2000; 12 : 2265-2272.

60. Strunk T, Inder T, Wang X, Burgner D, Mallard C, Levy O. Infection-induced inflammation and cerebral injury in preterm infants. Lancet Infect Dis 2014; 14: 751-762.

61. Eklind S, Mallard C, Arvidsson P, Hagberg H. Lipopolysaccharide induces both a primary and a secondary phase of sensitization in the developing rat brain. Pediatr Res 2005; 58 : 112-116.

62. Hickey E, Shi H, Van Arsdell G, Askalan R. Lipopolysaccharide-induced preconditioning against ischemic injury is associated with changes in toll-like receptor 4 expression in the rat developing brain. Pediatr Res 2011; 70: 10-14

63. Mallard C, Davidson JO, S T, Green CR, Bennet L, Robertson NJ et al. Astrocytes and microglia in acute cerebral injury underlying cerebral palsy associated with preterm birth. Pediatr Res 2014; 75: 234-240.

64. Connelly WM, Errington AC, Di Giovanni G, Crunelli V. Metabotropic regulation of extrasynaptic GABAA receptors. Front Neural Circ 2013; 7: 171.

65. Tanaka K. Alteration of second messengers during acute cerebral ischemia - adenylate cyclase, cyclic AMP-dependent protein kinase, and cyclic AMP response element binding protein. Prog Neurobiol 2001; 65: 173-207.

66. Muller MM, Middelanis J, Meier C, Surbek D, Berger R. 17beta-estradiol protects 7- day old rats from acute brain injury and reduces the number of apoptotic cells. Reprod Sci 2013; 20: 253-261.

67. Ramachandra R Atlas of the Neonatal Rat Brain. CRC Press: Boca Raton, London, 2011.

68. Ofengeim D, Chen YB, Miyawaki T, Li H, Sacchetti S, Flannery RJ et al. N-terminally cleaved Bcl-xL mediates ischemia-induced neuronal death. Nat Neurosci 2012; 15: $574-580$.

69. Jellema RK, Lima Passos V, Zwanenburg A, Ophelders DR, De Munter S, Vanderlocht J et al. Cerebral inflammation and mobilization of the peripheral immune system following global hypoxia-ischemia in preterm sheep. J Neuroinflammation 2013; 10: 13. 